\title{
Curvature Constraints from the Causal Entropic Principle
}

\author{
Brandon Bozek, Andreas Albrecht, and Daniel Phillips \\ Physics Department, University of California, Davis.
}

(Dated: October 26, 2018)

\begin{abstract}
Current cosmological observations indicate a preference for a cosmological constant that is drastically smaller than what can be explained by conventional particle physics. The Causal Entropic Principle (Bousso, et al.) provides an alternative approach to anthropic attempts to predict our observed value of the cosmological constant by calculating the entropy created within a causal diamond. We have extended this work to use the Causal Entropic Principle to predict the preferred curvature within the "multiverse". We have found that values larger than $\rho_{k}=40 \rho_{m}$ are disfavored by more than $99.99 \%$ and a peak value at $\rho_{\Lambda}=7.9 \times 10^{-123}$ and $\rho_{k}=4.3 \rho_{m}$ for open universes. For universes that allow only positive curvature or both positive and negative curvature, we find a correlation between curvature and dark energy that leads to an extended region of preferred values. Our universe is found to be disfavored to an extent depending the priors on curvature. We also provide a comparison to previous anthropic constraints on open universes and discuss future directions for this work.
\end{abstract}

PACS numbers:

\section{INTRODUCTION}

The simplest explanation for the observed acceleration of the universe is Einstein's cosmological constant, $\Lambda$. However, the value that explains the acceleration is many orders of magnitude smaller than that expected from quantum field theory. We are then left either to determine a method to set the cosmological constant to a small value or to consider $\Lambda$ an environmental variable varying from place to place in the multiverse.

Following the environmental approach numerous authors (many inspired by the pioneering work of Weinberg [1]), have sought to explain the observed value of $\Lambda$ by postulating that the most likely universe to be observed would be that which contains the largest potential to contain observers. However, such "anthropic" approaches can become burdened by complicated assumptions on the nature of observers. In their "Causal Entropic Principle" (CEP) Bousso et al. [2] took this reasoning in a simple and elegant direction by associating observers with entropy increase. Initial applications of this approach have successfully predicted our value of $\Lambda$ 2, 3]. The CEP has added appeal because there has been long standing (if not universal 4] ) acceptance of the idea that entropy increase would need to be imposed as a condition specific to observers rather than a global and eternal property of the Universe [5, 6, 7, 8]. Specifically, the CEP gives a weight to each set of cosmological parameters proportional to the entropy produced within a causal diamond in the corresponding cosmology. In addition to the original work[2] which found our value of $\Lambda$ to be within one sigma of the peak of their predicted probability distribution, the CEP was further developed by Cline et al. [3], exploring constraints on other cosmological values such as density contrast, baryon fraction, matter abundance, and dark matter annihilation rate.

In this paper we develop this method further by using CEP to jointly predict the values of curvature and $\Lambda$ most likely to be observed. Since Cline et al. [3] did not find significant features in their extended parameter space, we chose to vary only $\rho_{k}$ and $\rho_{\Lambda}$ for this work and hold all other parameters fixed. However, given the tail in the probability distribution for positive curvature, an interesting extension to this work would be to also vary additional parameters such as the density contrast.

Anthropic constraints on curvature are interesting in their own right, in the context of the "flatness problem" 9, 10] which suggests that in the absence of something like cosmic inflation [10, 11, 12] the "most natural" realization of big bang cosmology would be highly dominated by curvature. A number of authors have already considered anthropic bounds on curvature 13, 14, 15], but we believe this is the first work to apply the CEP to curvature. We find that our results place an upper limit on the allowed negative curvature as expected, but one that is looser than those works mentioned above due to a more lenient tolerance on the sizes of structure that are allowed to form. Further, we find that our peak probability for open universes to be away from the upper edge of our probability distribution, not dictated by it, as would be the case in the other work. We also consider solutions that allow for just positive curvature and for both positive and negative curvature. In these cases we find a tail in the probability distribution that allows for a wide range of allowed $\Lambda$ and $\rho_{k}$, a large fraction of which are significantly larger than our measured values. We find that our universe is not ruled out in any scenario, but, depending on one's choice of priors on curvature, disfavored to a certain degree.

In section II we review the CEP and the cosmology we will be considering. We then review the star formation model we will use in section III. We discuss our results in section IV and our conclusions in section V 

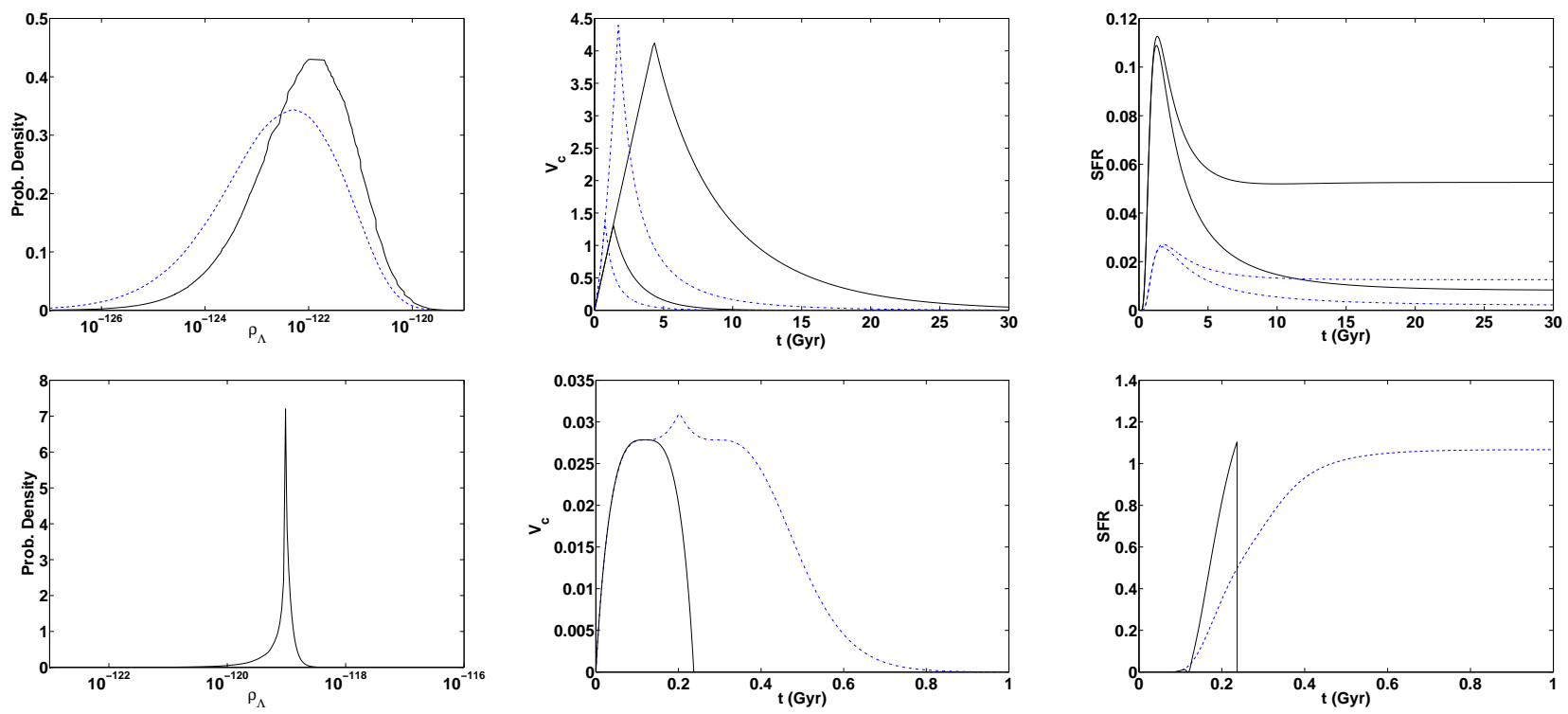

FIG. 1: Top Left Panel: Probability density for $\rho_{\Lambda}$ with fixed curvature of $\rho_{k}=10 \rho_{m}$ (dashed) and $\rho_{k}=0$ (solid). Top Center Panel: The comoving volume (in units of $10^{12} M p c^{3}$ ) for $\rho_{\Lambda}=10^{-123}$ (larger) and $\rho_{\Lambda}=10^{-122}$ (smaller) are shown for $\rho_{k}=10 \rho_{m}$ (dashed) and $\rho_{k}=0$ (solid). Top Right Panel: The star formation rate in units of $\frac{M_{\odot}}{M p c^{3} y r}$. The upper curve for each value of curvature represented in solid and dashed respectively is $\rho_{\Lambda}=10^{-122}$ and the lower is $\rho_{\Lambda}=10^{-123}$. The bottom row is the probability density, comoving volume, and star formation rate for $\rho_{k}=-50 \rho_{m}$. The blue/dashed curve is $\rho_{\Lambda}=10^{-119}$ and the black/solid curve is $\rho_{\Lambda}=10^{-120}$.

\section{THE CAUSAL ENTROPIC PRINCIPLE}

According to the CEP the probability distribution for $\Lambda$ is given by the equation:

$$
\frac{d^{2} P}{d \rho_{\Lambda} d \rho_{k}}=P_{0} \times w\left(\rho_{\Lambda}, \rho_{k}\right) \times \frac{d^{2} p}{d \rho_{\Lambda} d \rho_{k}}
$$

where $w\left(\rho_{\Lambda}, \rho_{k}\right)$ is a weighting factor, $P$ is the total probability, $P_{0}$ is a normalization factor, and $p$ is the total prior. We will assume the joint prior probability of $p\left(\rho_{\Lambda}, \rho_{k}\right)$ to be independent giving $p\left(\rho_{\Lambda}, \rho_{k}\right)=$ $p\left(\rho_{\Lambda}\right) \times p\left(\rho_{k}\right)$. The prior for $\Lambda$ is an expression of how the "multiverse" is populated by physics with different values of $\Lambda$. Here we use the standard form (sometimes motivated by the string theory landscape) taken by previous authors [1, 2]: $\frac{d p}{d \rho_{\Lambda}}=$ constant. For simplicity we also take $\frac{d p}{d \rho_{k}}=$ constant, which will enable a discussion of the flatness problem later in the paper. These "flat" priors mean the largest allowed "cutoff" values of $\rho_{k}$ and $\rho_{\Lambda}$ set the typical values for the prior. The value of the cutoff turns out to be unimportant because for flat priors $w\left(\rho_{\Lambda}, \rho_{k}\right)$ dictates the shape of the final probability distribution.

In the CEP framework we set $w\left(\rho_{\Lambda}, \rho_{k}\right)=\Delta S$, where $\Delta S$ is the total entropy produced within a causal diamond. After considering numerous astrophysical sources for entropy production, Bousso et al. [2] find that the dominant form of entropy production is star light reradi- ated by dust. As in [2], $\Delta S$ is given by.

$$
\Delta S=\int_{t_{i}}^{\infty} \frac{d^{2} S}{d V_{c} d t} V_{c} d t
$$

where $V_{c}$ is the total comoving volume of an observer's causal patch. A causal patch is defined by a future light cone taken at an initial point, such as reheating following inflation, intersected by a past light cone at a late time point, which in the case of a universe dominated by a cosmological constant is bounded by a de Sitter horizon and in the case of a universe dominated by positive curvature the late time event is the crunch.

We use the metric:

$$
d s^{2}=-d t^{2}+a(t)^{2} R_{0}^{2}\left[d \chi^{2}+S_{k}(\chi)^{2} d \Omega^{2}\right]
$$

where $S_{k}(\chi)=\sin (\chi)$ for positive curvature, $S_{k}(\chi)=$ $\sinh (\chi)$ for negative curvature, and $S_{k}(\chi)=\chi$ for no curvature. The causal diamond is then given by $R_{0} \chi=$ $\frac{\Delta \tau}{2}-\left|\frac{\Delta \tau}{2}+\tau\right|$, where $\tau=\int \frac{d t}{a(t)}$. The comoving volume is then:

$$
V_{c}= \begin{cases}2 \pi R_{0}^{3}\left[\chi-\frac{1}{2} \sin (2 \chi)\right] & \text { for } \mathrm{k}=+1 \\ \frac{4 \pi}{3} R_{0}^{3} \chi^{3} & \text { for } \mathrm{k}=0 \\ 2 \pi R_{0}^{3}\left[\frac{1}{2} \sinh (2 \chi)-\chi\right] & \text { for } \mathrm{k}=-1\end{cases}
$$

The scale factor $a(t)$ can be found by solving the Friedmann equation:

$$
H^{2}=\frac{8 \pi}{3}\left(\frac{\rho_{m}}{a^{3}}+\rho_{\Lambda}+\frac{\rho_{k}}{a^{2}}\right)
$$


where $\rho_{\Lambda}=\Lambda / 8 \pi, \rho_{k}=-3 k / 8 \pi R_{0}^{2}$, and $k=\{-1,0,1\}$ for a negative, flat, and positively curved universe respectively. The value of the matter density today $(a=1)$ is set at $\rho_{m}=5.2 \times 10^{-124}$ in Planck units which we use throughout unless otherwise noted. Following previous work in the topic we neglect radiation. In this work we hold $\rho_{m}$ fixed and allow the curvature today, $\rho_{k}$, and $\rho_{\Lambda}$ to vary.

The other part of Eqn. 2, $\frac{d^{2} S}{d V_{c} d t}$, is the entropy produced per comoving volume per time, which is calculated by the convolution

$$
\frac{d^{2} S}{d V_{c} d t}(t)=\int_{0}^{t} \frac{d^{2} S}{d M d t^{\prime}}\left(t-t^{\prime}\right) \dot{\rho_{\star}}\left(t^{\prime}\right) d t^{\prime}
$$

where $\frac{d^{2} S}{d M d t^{\prime}}\left(t-t^{\prime}\right)$ is the entropy production rate per stellar mass at time $t$ due to stars born at an earlier time, $t^{\prime}$, and $\dot{\rho}_{\star}\left(t^{\prime}\right)$ is the star formation rate at $t^{\prime}$. The entropy rate per stellar mass is found by calculating

$$
\frac{d^{2} S}{d M d t^{\prime}}\left(t-t^{\prime}\right)=\frac{1}{\langle M\rangle} \int_{0.08 M_{\odot}}^{M_{\max }\left(t-t^{\prime}\right)} \frac{d^{2} s}{d N_{\star} d t} \xi_{I M F}(M) d M
$$

where $\xi_{I M F}(M)$ is the initial mass function and $\frac{d^{2} s}{d N_{\star} d t}$ is the entropy production rate for a single star. The latter is given by the stellar luminosity divided by the effective temperature. The number of photons emitted by a star is dominated by the half that are reprocessed by dust at an effective temperature of $20 \mathrm{mev}$. This is given by:

$$
\frac{d^{2} s}{d N_{\star} d t}=\frac{L_{\star}}{T_{e f f}}=\frac{1}{2}\left(\frac{M}{M_{\odot}}\right)^{3.5} 3.7 \times 10^{54} y^{-1} .
$$

The prefactor in Eqn. 7 is the average initial mass, $\langle M\rangle=0.48 M_{\odot}$. The lower limit of Eqn. 7 is the minimum mass of a star that can support nuclear burning. Following Bousso, we take the upper limit to be

$$
M_{\text {max }}\left(t-t^{\prime}\right)= \begin{cases}100 M_{\odot} & \text { for } t-t^{\prime}<10^{5} \mathrm{yr} \\ \left(\frac{10^{10} y r}{t-t^{\prime}}\right)^{0.4} M_{\odot} & \text { for } t-t^{\prime}>10^{5} \mathrm{yr}\end{cases}
$$

\section{STAR FORMATION}

A key aspect to this work is how well the star formation rate is modeled. While Bousso et al. [2] use star formation rates of Nagamine [16] et al. and Hopkins and Beacom [17] with some simple modifications to extend these models to include different cosmological constant values, we will follow Cline et al. [3] who use a model proposed by Hernquist and Springel (HS) [18. The HS model was found to produce similar results to those found in Bousso et al. but was more straightforward to extrapolate to the case were a larger number cosmological parameters are varied. The HS star formation model is given by this equation:

$$
\dot{\rho}_{\star}=\rho_{m} s_{0} q(t)\left(1-\operatorname{erf}\left(\sqrt{\frac{a}{2}} \frac{\delta_{c}}{\sigma_{4}}\right)\right)
$$

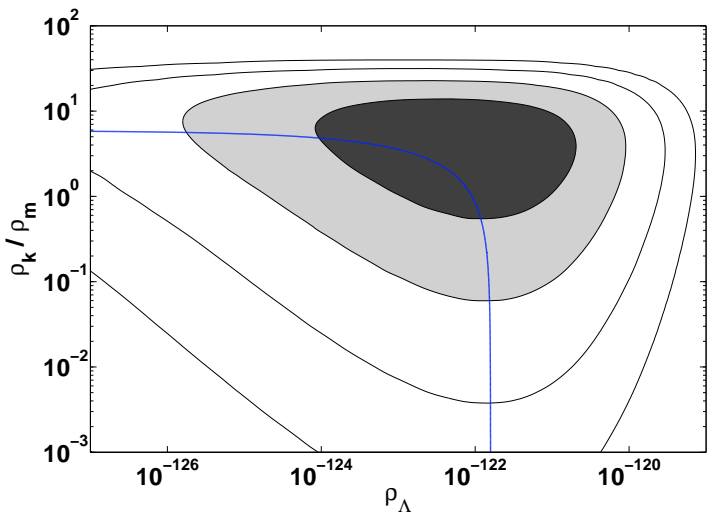

FIG. 2: The $68.27 \%$ (dark grey), $95.44 \%$ (light grey), $99.73 \%$ (inner white), and $99.99 \%$ (outer white) contours for a multiverse that only admits negative curvature universes. The solid blue line that cuts through the other 4 contours is the anthropic bound from [15] for smaller sized galaxies.

where $\delta_{c}=1.6868, a=0.707, s_{0}=3.7995 \times 10^{-63}$ (taken from [18]), and $\rho_{m}$ (same as above) are constants and $\sigma_{4}$ is the root-mean-square density fluctuation that corresponds to the mass scale that virializes at a temperature of $T=10^{4} \mathrm{~K}$.

The star formation efficiency, $q(t)$, encompasses the rate and efficiency of radiative cooling within a collapsing object that leads to star formation. HS model this process with:

$$
q(t)=\left(\frac{\chi(t) \tilde{\chi}}{\left(\chi(t)^{m}+\tilde{\chi}^{m}\right)^{\frac{1}{m}}}\right)^{p}
$$

where $\chi(t)=\left(\frac{H}{H_{0}}\right)^{2 / 3} . \quad \tilde{\chi}=4.6, m=6$, and $p=2.72$ are constants fit from numerical simulations and $H_{0}=$ $70 \frac{\mathrm{km} / \mathrm{s}}{\mathrm{Mpc}}$. For universes with positive curvature that end in a crunch, the star formation rate of Eqn. 10 continues up until the crunch. We therefore needed to place a bound on late time star formation when we no longer trust our model. We set $\dot{\rho}_{\star}=0$ when $\rho_{r}=\rho_{m}$ in the collapsing phase, where $\rho_{r}=1.5 \times 10^{-127}$. This choice allows for an exploration of the CEP properties without a strong limiting effect put in by hand.

Star formation rates for several different values of curvature and $\Lambda$ are shown in the right panel of Fig. 1, Their corresponding probability curves are shown in the left panel of Fig. 1

\section{RESULTS AND DISCUSSION}

Using the equations of the previous section we solve for the probability distribution over a wide range of open and closed universes. To enable the most general discussion we consider both open and closed cosmologies together and separately. (It is commonly [15] but not universally [19] thought that the string theory landscape only leads to the open case.) 


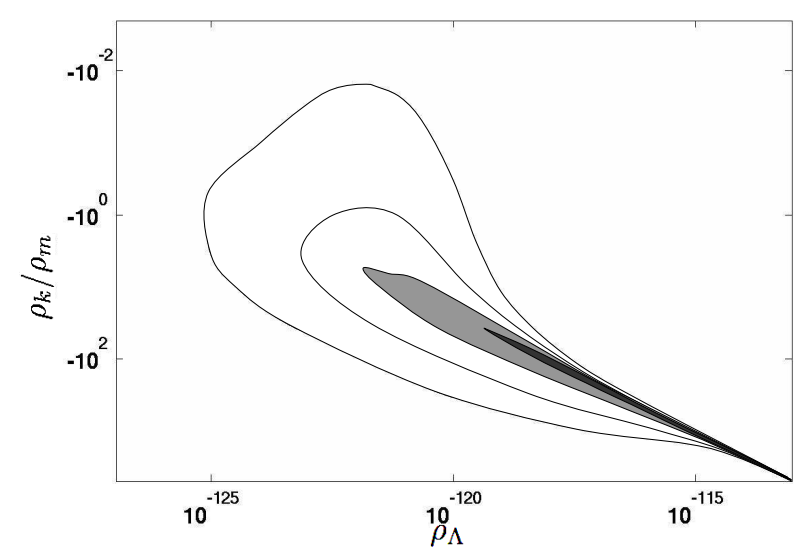

FIG. 3: The $68.27 \%$ (dark grey), $95.44 \%$ (light grey), $99.73 \%$ (inner white), and $99.99 \%$ (outer white) contours for a multiverse that only admits positive curvature universes.

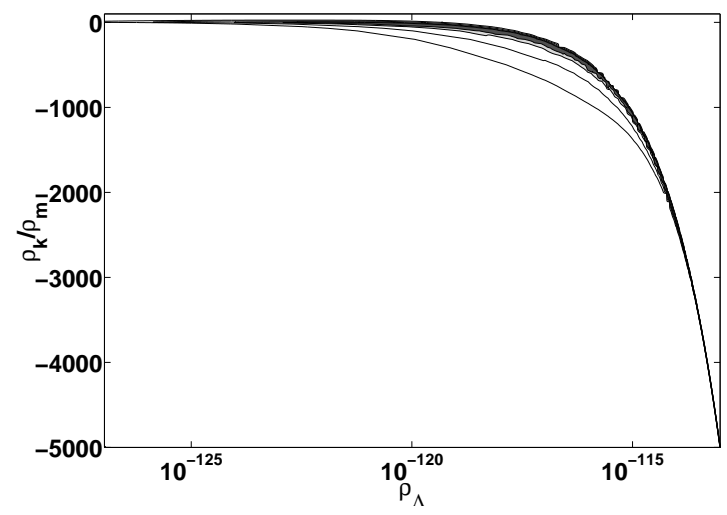

FIG. 4: The $68.27 \%$ (dark grey), $95.44 \%$ (light grey), $99.73 \%$ (inner white), and $99.99 \%$ (outer white) contours for a multiverse that admits both positive and negative curvature universes.

If we were to only consider open universes, then Fig. 2 depicts the resulting probability density distribution in $\log \rho_{\Lambda}-\log \rho_{k}$ space. Values of curvature of $\rho_{k}>40 \rho_{m}$ fall outside of the $99.99 \%$ CL. Smaller values of $\Lambda$ and curvature lead to larger causal diamonds and therefore have the most total entropy production, as depicted in Fig. 1. This balances the majority of vacua having larger values of both $\Lambda$ and curvature, giving a peak value at $\rho_{\Lambda}=7.9 \times 10^{-123}$ and $\rho_{k}=4.3 \rho_{m}$. Using the upper bound $(95 \% \mathrm{CL})$ on negative curvature from WMAP + HST [20], our universe of $\rho_{\Lambda}=1.25 \times 10^{-123}$ and $\rho_{k}=0.016 \rho_{m}$ is in the $99.73 \%$ CL. Fig. 1 illustrates that for a fixed value of negative curvature the distribution for $\Lambda$ remains roughly unchanged from the distribution for a flat universe.

Now considering only positively curved universes, there is a clear correlation between $\Lambda$ and curvature which comes from competition between positive curvature and $\Lambda$. This can cause the universe to "loiter" 21] in a state with little cosmic expansion but plenty of structure growth. These conditions conspire to create both a larger causal diamond and enhanced linear growth. This results in the tail on the bottom right of Fig. 3 where there is ridge between collapsing regions to the left and non-collapsing regions to the right in the $68 \%$ CL. For a fixed value of positive curvature, small values of $\Lambda$ lead to a universe that will recollapse before there is significant star formation. As $\Lambda$ is increased the recollapse is delayed allowing for more star formation and a larger causal diamond, giving more total entropy produced and therefore a more likely universe within the CEP framework. This continues until $\Lambda$ is large enough to allow for a non-collapsing universe, at which point larger values of $\Lambda$ begin to suppress growth. The narrowing of the tail comes from deviations from the ridge having large energy densities that lead to either a rapid recollapse or early $\Lambda$ domination. Using the upper bound $(95 \% \mathrm{CL})$ on positive curvature from WMAP+HST [20], our universe of $\rho_{\Lambda}=1.25 \times 10^{-123}$ and $\rho_{k}=-0.06 \rho_{m}$ is in the $99.99 \%$ CL.

Fig. 4- 4 shows the 2 dimensional probability density distribution, $\frac{d^{2} P}{d \log \rho_{\Lambda} d \rho_{k}}$, in $\log \rho_{\Lambda^{-}} \rho_{k}$ space for both positive and negative curvature. The full span range of curvature allowed by WMAP+HST [20] $\left(-0.06 \rho_{m} \leq \rho_{k} \leq\right.$ $\left.0.016 \rho_{m}\right)$ is in the $95.44 \%$ CL. A significant fraction of the values within the $68.27 \%$ CL are positively curved universes of both large amounts of curvature and dark energy compared with our universe due to the competing effects mentioned above leading to a similar tail on the lower right.

A recent paper by Bousso and Leichenauer [22] has argued that the asymptotic behavior of the star formation model shown in upper right panel of Fig. 1 may be unphysical. Since the CEP framework depends on an accurate accounting of star formation in universes far different than ours, a careful study of different models is an important aspect of developing this work further. However, we suspect that the asymptotic behavior leads to a subdominant effect on the final probability distributions since it coincides with a decreasing comoving volume that will diminish the contribution it will make to the total entropy contribution.

On the other hand, the bottom right portion of the tail in Figs. 3 and 4 is an area where we have little confidence in our star formation model as the duration of matter domination is increasingly smaller as we move further out onto the tip of the distribution. A different cut on late time star formation from the one we chose above or another star formation model may find the bottom right tip less favored or ruled out.

We have extended the CEP to include curvature and found that regardless of whether one considers both positive and negative curvature or just one of the two options, a non-zero value of curvature appears to be preferred. We have also found that our universe is not ruled out in any 
scenario considered here, but is somewhat disfavored in some scenarios. The favored values for an open universe are just a few orders of magnitude larger than values favored by modern data, so to the extent that the flatness puzzle is about why the curvature is not given by the Planck scale, the CEP seems to put a significant dent in the flatness puzzle. This is not dissimilar to anthropic arguments of curvature, where structure formation is cut off by excessive curvature, however the CEP offers a less restrictive initial assumption. In Fig. 2 we also plot the bound on negative curvature calculated by Freivogel et al. 15. (which are similar to those of Vilenkin and Winitzki [13] and Garriga et al. 14]) by demanding that structures at least as large as a small sized galaxy form. Our plot allows for somewhat more curvature than is allowed by these methods. Setting a structure formation limit based on smaller galactic masses brings the curvature limit closer to ours. Ultimately our rough reproduction of the anthropic cutoff is unsurprising as our main entropy source, star formation, cuts off along with structure formation. However, our actual prediction for curvature is not against a cutoff for structure formation as would be the case for a simple bound. The causal entropic weighting provides additional rewards for smaller curvatures in the form of increased star formation and entropy production.

\section{CONCLUSIONS}

Anthropic constraints on observable parameters are interesting when considering implications for the multi- verse. The CEP has appealing advantages over previous anthropic attempts. We find that the CEP places upper limits on the amount of curvature that is observable and while not ruling out our universe, the CEP finds larger curvature preferable to our measured value. We also find an intriguing feature in the probability space for positive curvature of an elongated tail stretching into regions of large curvature. Our results for negatively curved universes are broadly consistent with previous anthropic bounds on curvature but less constraining due to a more lenient tolerance for the minimum mass of structure allowed. Still, like the previous work we find that anthropic considerations seem to offer cosmology considerable relief from the flatness problem.

\section{Acknowledgments}

We thank Lloyd Knox, Damien Martin, and especially James Cline for very helpful discussions. We also thank Tony Tyson for computing resources as well as Perry Gee and Jim Bosch for technical computing support. This work was supported by DOE grant DE-FG0391ER40674.
[1] S. Weinberg, Phys. Rev. Lett. 59, 2607 (1987).

[2] R. Bousso, R. Harnik, G. D. Kribs, and G. Perez, Phys. Rev. D76, 043513 (2007), hep-th/0702115.

[3] J. M. Cline, A. R. Frey, and G. Holder, Phys. Rev. D77, 063520 (2008), 0709.4443.

[4] R. P. Feynman, The character of physical law [by] Richard Feynman (M.I.T. Press Cambridge,, 1965).

[5] L. Boltzmann, Nature (London) 51, 413 (1895).

[6] A. Albrecht (2002), astro-ph/0210527.

[7] L. Dyson, M. Kleban, and L. Susskind, JHEP 10, 011 (2002), hep-th/0208013.

[8] A. Albrecht and L. Sorbo, Phys. Rev. D70, 063528 (2004), hep-th/0405270.

[9] R. H. Dicke and P. J. E. Peebles, in GENERAL RELATIVITY. AN EINSTEIN CENTENARY SURVEY, edited by S. W. Hawking and W. Israel (????), cambridge, United Kingdom: Univ.Pr.(1979) 919p.

[10] A. H. Guth, Phys. Rev. D23, 347 (1981).

[11] A. D. Linde, Phys. Lett. B108, 389 (1982).

[12] A. Albrecht and P. J. Steinhardt, Phys. Rev. Lett. 48,
1220 (1982).

[13] A. Vilenkin and S. Winitzki, Phys. Rev. D55, 548 (1997), astro-ph/9605191.

[14] J. Garriga, T. Tanaka, and A. Vilenkin, Phys. Rev. D60, 023501 (1999), astro-ph/9803268.

[15] B. Freivogel, M. Kleban, M. Rodriguez Martinez, and L. Susskind, JHEP 03, 039 (2006), hep-th/0505232.

[16] K. Nagamine, J. P. Ostriker, M. Fukugita, and R. Cen, Astrophys. J. 653, 881 (2006), astro-ph/0603257.

[17] A. M. Hopkins and J. F. Beacom, Astrophys. J. 651, 142 (2006), astro-ph/0601463.

[18] L. Hernquist and V. Springel, Mon. Not. Roy. Astron. Soc. 341, 1253 (2003), astro-ph/0209183.

[19] R. V. Buniy, S. D. H. Hsu, and A. Zee, Phys. Lett. B660, 382 (2008), hep-th/0610231.

[20] E. Komatsu et al. (WMAP) (2008), 0803.0547.

[21] V. Sahni, H. Feldman, and A. Stebbins, Astrophys. J. 385, 1 (1992).

[22] R. Bousso and S. Leichenauer (2008), 0810.3044. 